\title{
Brisk walking and serum lipid and lipoprotein variables in previously sedentary women - effect of 12 weeks of regular brisk walking followed by 12 weeks of detraining
}

\author{
Adrianne E. Hardman PhD and Annette Hudson MSc \\ Department of Physical Education, Sports Science and Recreation Management, Loughborough University, \\ Loughborough, UK
}

\begin{abstract}
The purpose of this study was to examine the effectiveness of brisk walking as a means of improving endurance fitness and influencing serum lipid and lipoprotein variables in previously sedentary women. Walkers $(n=10$, mean(s.e.m.) age 47.3(2.0) years) followed a programme of brisk walking (mean(s.e.m.) speed $1.76(0.03) \mathrm{m} \mathrm{s}^{-1}$ ) for 12 weeks, after which the training stimulus was withdrawn. Controls $(n=10$, mean(s.e.m.) age 41.6(1.2) years) maintained their habitual sedentary lifestyle throughout. Endurance fitness was determined using laboratory measures of responses to treadmill walking. Serum lipid and lipoprotein variables were determined in venous blood (12-h fasted). Body fatness was assessed by anthropometry and dietary practice using the 7-day weighed food intake technique. Measurements were repeated after 12 and 24 weeks. Brisk walking resulted in a decrease in heart rate and blood lactate concentration during exercise, while detraining was accompanied by a reversal of these changes. Changes in body mass and the ratio of circumferences at the waist and hip did not differ between groups but the sum of four skinfolds decreased with brisk walking and increased with detraining. High density lipoprotein (HDL) and $\mathrm{HDL}_{2}$ cholesterol increased with walking and decreased with detraining but no between group changes (analysis of variance, $P<0.05$ ) were found in other lipid and lipoprotein variables. These findings suggest that regular brisk walking can improve endurance fitness and increase HDL cholesterol concentration in sedentary women.
\end{abstract}

Keywords: Walking, women, HDL cholesterol, endurance fitness

Coronary heart disease (CHD) is an important cause of morbidity and mortality in women as well as in men and yet knowledge about means of modifying cardiovascular risk factors in women is surprisingly limited. Arguably, the most important lipid risk factor in women is the blood concentration of high density lipoprotein (HDL) cholesterol ${ }^{1}$. Several

Address for correspondence: Dr A. E. Hardman, Department of Physical Education, Sports Science and Recreation Management, Loughborough University, Loughborough, Leicestershire LE11 3TU, UK

(C) 1994 Butterworth-Heinemann Ltd 0306-3674/94/040261-06 epidemiological studies have found it to be a strong, negative, independent predictor of CHD in women, an increase of $0.26 \mathrm{mmoll}^{-1}$ being associated with a $42-50 \%$ decrease in risk ${ }^{2,3}$. One positive influence on HDL cholesterol may be physical activity as endurance trained women are reported to have concentrations which are nearly $30 \%$ higher than in comparable sendentary women ${ }^{4}$. Despite the consistency of cross-sectional studies there is conflicting evidence about whether or not increased levels of physical activity result in an elevated HDL concentration in women $^{5-8}$ and, if this seems to be the case, about what type and amount of exercise is effective.

In a previous study we reported that regular brisk walking resulted in a marked increase in plasma HDL cholesterol $^{9}$. The purpose of the present study was to examine more rigorously the hypothesis that brisk walking - performed in a flexible, self-governed programme - is a sufficient exercise stimulus to influence serum lipid and lipoprotein variables in formerly sendentary women. The approach adopted was to monitor these variables during a 12-week exercise programme and a subsequent 12-week period during which the training stimulus was withdrawn.

\section{Subjects and methods}

Twenty women volunteered to take part in this study, which had the approval of the University's Ethical Advisory Committee. Each subject completed a physical activity questionnaire (maximum score 200) concerned with their exercise habits over the past 10 years. Responses showed that no individual had engaged in regular exercise during the 2 years before the study (mean(s.e.m.) scores = walkers 42 (3), controls 46(4); analysis of variance, $P<0.05$, not significantly different). Subjects were allocated into groups (walkers and controls) on a convenience basis - walkers followed a progressive training programme of brisk walking for the first 12 weeks of the study, and returned to their previous sedentary lifestyle for the second 12 weeks. Controls were asked to maintain their habitual lifestyle throughout the whole period of the study. Walkers and controls were of mean(s.e.m.) age 47.3(2.0) years and 41.6(1.2) years, 
respectively, with mean(s.e.m.) body mass indices of $24.8(1.4) \mathrm{kg} \mathrm{m}^{-2}$ and $23.4(1.1) \mathrm{kg} \mathrm{m}^{-2}$. All measurements were made at base line and repeated after 12 and 24 weeks.

\section{Exercise tests}

At the start of the study only, each subject completed a 1.6- $\mathrm{km}$ walk around an athletics track at a pace she judged to be 'brisk'. Two laboratory exercise tests were conducted at each observation point: (1) a $1.6-\mathrm{km}$ walk on a motorized treadmill at the pace selected by each individual outdoors; and (2) an incremental, uphill treadmill walking test. The latter involved walking at a standard speed $\left(1.34 \mathrm{~m} \mathrm{~s}^{-1}\right)$ for four consecutive, 4-min periods. At the end of each $4 \mathrm{~min}$ the gradient was increased, these being selected to elicit $50,60,70$ and $80 \%$ of each individual's predicted maximal oxygen uptake - as determined from steady state measurements of oxygen uptake and heart rate during a preliminary test ${ }^{10}$. During both treadmill tests expired air samples were collected using standard Douglas bag techniques and analysed to determine oxygen uptake and carbon dioxide production. Heart rate was measured from the electrocardiogram (modified Lead I). Capillary blood samples $(20 \mu \mathrm{l}$ in duplicate) were obtained before each test, at the end of the $1.6-\mathrm{km}$ walk and at the end of each stage of the incremental test. These were immediately deproteinized and stored at $-20^{\circ}$ Celsius before analysis for lactate ${ }^{11}$. Data from the incremental test were used to interpolate the oxygen uptake $\left(\dot{V}^{\mathrm{O}_{2}}\right)$ at a reference blood lactate concentration of $2 \mathrm{mmoll}^{-1}$, a sensitive index of endurance fitness which could be measured during submaximal exercise without extrapolation.

\section{Blood biochemistry}

Subjects attended the laboratory in the morning, after a 12-h fast and a 10-ml blood sample was obtained by venipuncture without stasis, after the subject had been seated for $10 \mathrm{~min}$. Blood was separated and $\mathrm{HDL}$ cholesterol and the cholesterol in the $\mathrm{HDL}_{3}$ subfraction were determined in fresh serum (stored at $5^{\circ}$ Celsius for $\leqslant 3$ days) after selective precipitation procedures using heparin-manganese and dextran sulphate ${ }^{12,13}$. $\mathrm{HDL}_{2}$ values were determined by subtraction of $\mathrm{HDL}_{3}$ from total HDL. Serum was stored at $-70^{\circ}$ Celsius before analysis for total cholesterol by a cholesterol oxidase method (Boehringer Mannheim, Mannheim, Germany), triacylglycerol by hydrolysis (Boehringer Mannheim) and apolipoproteins A-1 and B (Apo A-1, Apo B) by immunoprecipitin analysis (Atlantic Antibodies, Winnersh, UK). Imprecision was evaluated using standard sera (Precinorm, Boehringer Mannheim) and day-to-day coefficients of variation were: $<3.2 \%$ and $<3.6 \%$ for total and HDL cholesterol respectively; $4.5 \%$ for triglycerides; and $<2.4 \%$ and $<1.8 \%$ for Apo A-1 and Apo B, respectively.

\section{Anthropometry}

Height and body mass were determined and the amount and distribution of body fat was evaluated by measuring skinfold thicknesses at four standard sites, namely biceps, triceps, subscapular and suprailiac, as well as the ratio of circumferences at the waist and hip $^{14}$. All skinfold measurements were made by the same experienced observer at each stage of the study, using previously calibrated calipers (Holtain).

\section{Weighed food intake}

Average daily intakes of energy, fat and alcohol were assessed, together with the ratio of polyunsaturated to saturated fat, by the 7-day weighed food intake method with subsequent analysis using a computerized version (Salford Microdiet, Department of Mathematics and Computer Science, University of Salford, Salford, UK) of food composition tables ${ }^{15}$.

\section{Exercise programme}

After completion of the base line tests the walkers undertook a progressive, unsupervised 12-week programme of brisk walking. A minimum exercise prescription was provided which increased from $180 \mathrm{~min}$ in each of the first 2 weeks to $315 \mathrm{~min}^{\text {week }}{ }^{-1}$ during weeks 9-12. After initial advice given on an individual basis, subjects trained at their own brisk pace, the only constraints imposed being: (1) no training walk was to be shorter than $20 \mathrm{~min}$; and (2) the total training time had to be achieved by completing at least three sessions each week. A training diary describing the duration and frequency of the sessions was submitted by each walker every 2 weeks.

\section{Statistical analysis}

The response over time was compared between walkers and controls using two way analysis of variance for repeated measures, adopting a $5 \%$ level of confidence. Walkers were older than controls and therefore age was used as a fixed covariate. Multivariate criteria were employed (Statistica Mac, Statsoft, Tulsa, Oklahoma, USA; Release 3) to test the significance of differences between cell means when the response over time differed between groups.

\section{Results}

Mean(s.e.m.) self-selected brisk walking speed was $1.76(0.03) \mathrm{m} \mathrm{s}^{-1}$ and $1.77(0.04) \mathrm{m} \mathrm{s}^{-1}$ for walkers and controls, respectively. When the outdoor walking test was replicated on the treadmill, the same brisk walking speed elicited mean(s.e.m.) oxygen uptake values of $18.3(0.8) \mathrm{ml} \mathrm{kg}^{-1} \mathrm{~min}^{-1}$ and $18.6(0.6) \mathrm{ml}$ $\mathrm{kg}^{-1} \min ^{-1}$ and mean(s.e.m.) heart rates of $130(6)$ beat $\min ^{-1}$ and 128(5) beat $\min ^{-1}$, for walkers and controls respectively. The mean(s.e.m.) amount of brisk walking accomplished increased progressively from $187(6)$ min during each of the first 2 weeks to 292(7) min per week for the last 6 weeks.

Changes in the blood lactate and heart rate responses to exercise differed significantly over time between walkers and controls. Brisk walking resulted in decreases in the heart rate and blood lactate response to uphill treadmill walking (Figures 1 and 2) 


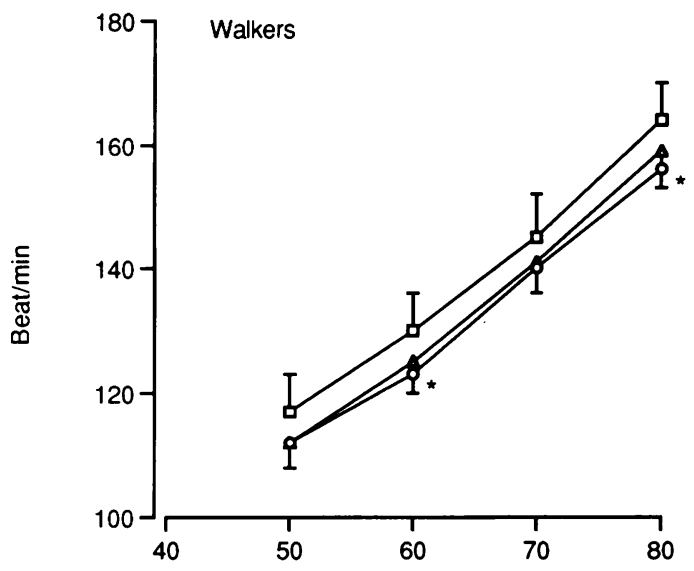

a

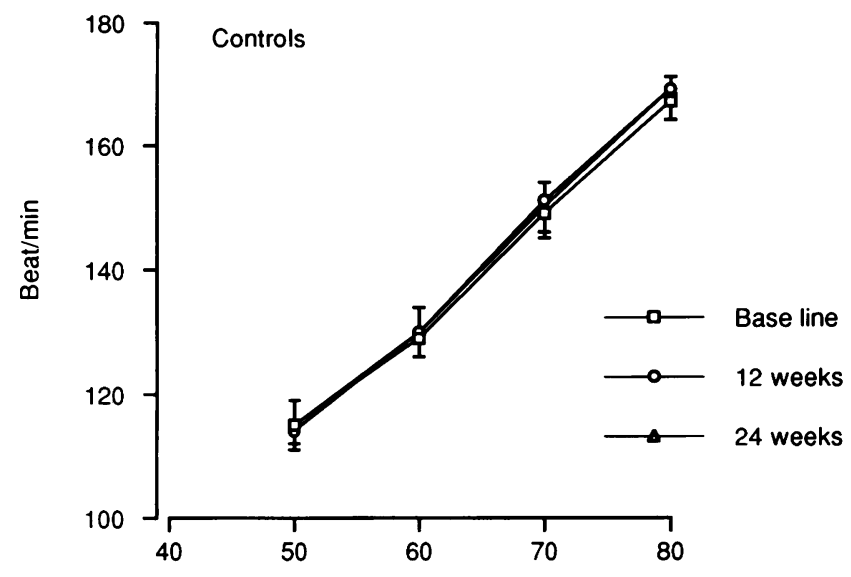

b

$\%$ predicted maximal oxygen uptake

Figure 1. Changes in heart rate during uphill treadmill walking for walkers and controls. Values are mean(s.e.m.); *significantly different from baseline, $P<0.05$

and an increase in the oxygen uptake at a reference blood lactate concentration, our measure of endurance fitness (Figure 3). When brisk walking training was discontinued these adaptations were reversed, the metabolic effect of detraining, as assessed by blood lactate concentration, being more marked than the effect on heart rate.

There were no between group differences in the changes in body mass or the ratio of circumferences at the waist and hip but changes in the sum of four skinfolds differed between walkers and controls, decreasing with brisk walking and increasing with detraining (Table 1). Values for serum lipoprotein variables are shown in Table 2. Total cholesterol (TC) concentration decreased over time but this response did not differ between groups. Both $\mathrm{HDL}$ and $\mathrm{HDL}_{2}$ cholesterol changed in a different way in walkers and controls - for both variables, an increase was apparent with brisk walking and a decrease with subsequent detraining. Controlling for accompanying changes in fatness, by including the sum of

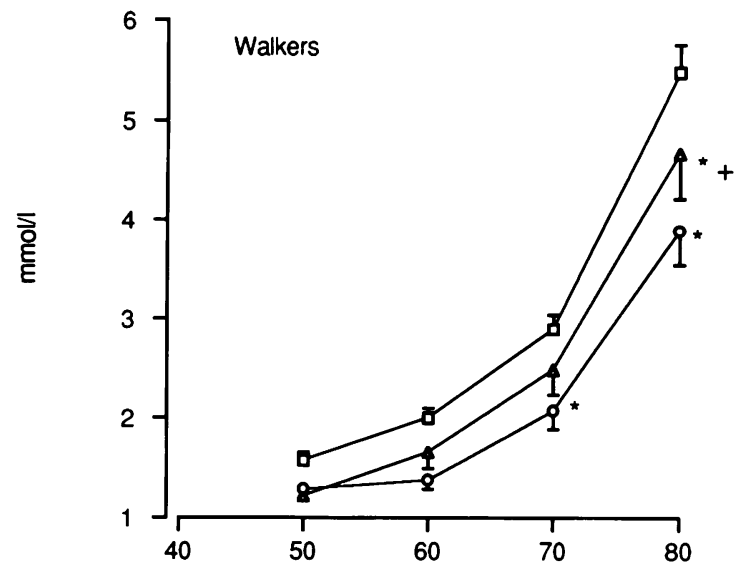

a

$\%$ predicted maximal oxygen uptake

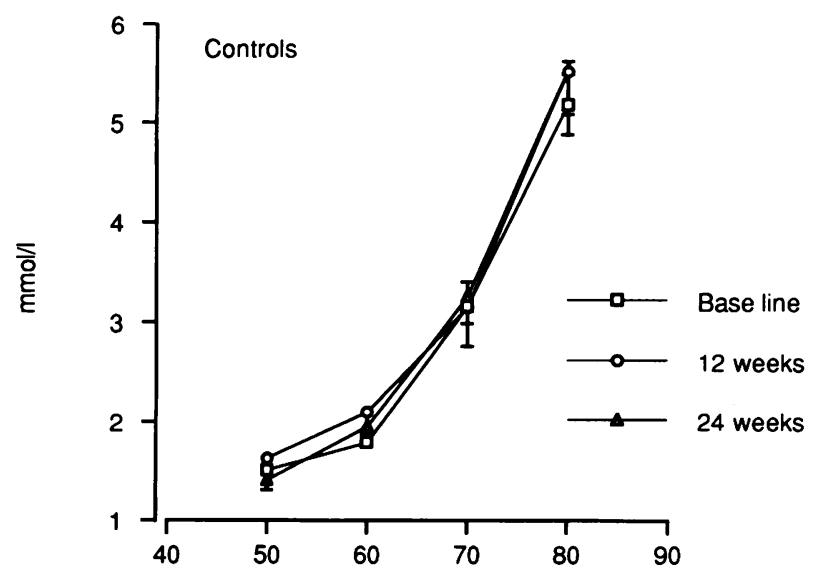

b $\%$ predicted maximal oxygen uptake

Figure 2. Changes in blood lactate concentration during uphill treadmill walking for walkers and controls. Values are mean(s.e.m.); *significantly different from baseline, $P<0.05 ;+$ significantly different from 12 weeks, $P<0.05$

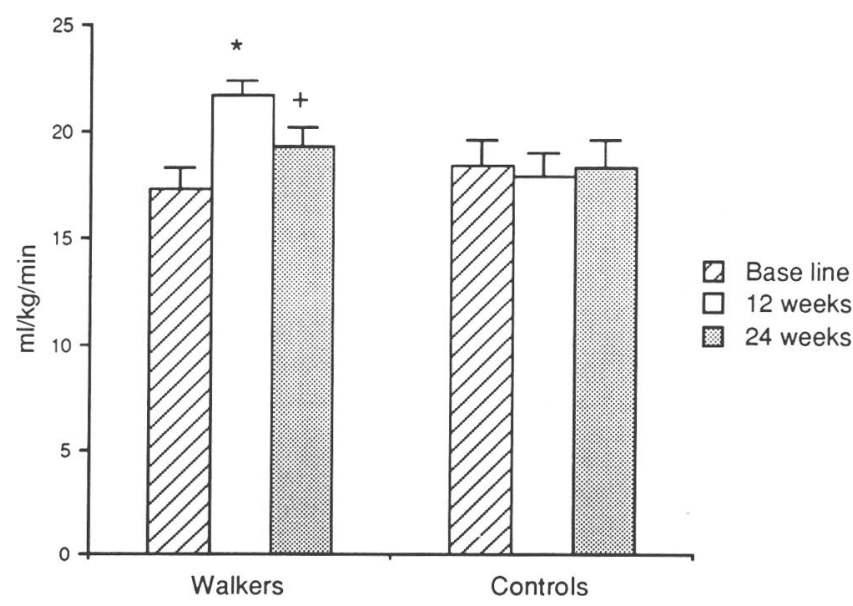

Figure 3. Changes in the oxygen uptake at a reference blood lactate concentration of $2 \mathrm{mmoll}^{-1}$ for walkers and controls. Values are mean(s.e.m.); *significantly different from baseline, $P<0.05 ;+$ significantly different from 12 weeks, $P<0.05$ 
skinfolds as a changing covariate, removed the between group difference in the changes in $\mathrm{HDL}_{2}$ cholesterol but not in HDL cholesterol. Despite the changes in $\mathrm{HDL}$ and $\mathrm{HDL}_{2}$ cholesterol, there was no influence of brisk walking on serum triacylglycerol concentration or apolipoproteins A-1 or B.

There were no between group differences in total energy intake over time, nor were there differences in the proportion of energy from dietary fat or the ratio of polyunsaturated to saturated fat in the diet (Table $3)$.

\section{Discussion}

The main finding of this study is that, in this group of formerly sedentary women, serum concentrations of $\mathrm{HDL}$ and $\mathrm{HDL}_{2}$ cholesterol increased with brisk walking and decreased when the training stimulus was removed. Moreover, these changes and the associated improvements in endurance fitness resulted from a flexible, home-based programme of moderate intensity exercise. An estimate of the distance walked can be made from the training records of the walkers; assuming that brisk walking pace approximated that determined during the track walk $\left(1.76 \mathrm{~m} \mathrm{~s}^{-1}\right.$ or about $15 \mathrm{~min} 38 \mathrm{~s}$ per mile), the distance covered was about $19 \mathrm{~km}$ in the first week, rising to about $30 \mathrm{~km}$ per week over each of the last 6 weeks. No injuries were reported, probably because of the nature of the exercise. Mean(s.e.m.) heart rates during brisk walking $\left(130(6)\right.$ beat $\left.\mathrm{min}^{-1}\right)$ represented some $72(3) \%$ of predicted maximal heart rates, values which would be expected to elicit a training effect ${ }^{16}$.

The effectiveness of the training programme was confirmed by the marked changes in the heart rate and blood lactate response to standardized exercise.

Table 1. Body mass, sum of four skinfolds and the ratio of circumferences at the waist and hip for walkers and controls

\begin{tabular}{|c|c|c|c|c|c|c|}
\hline & \multicolumn{3}{|c|}{ Walkers $(\mathrm{n}=10)$} & \multicolumn{3}{|c|}{ Controls $(n=10)$} \\
\hline & Base line & 12 weeks & 24 weeks & Base line & 12 weeks & 24 weeks \\
\hline $\begin{array}{l}\text { Body mass }(\mathrm{kg}) \\
\sum \text { four skinfolds }(\mathrm{mm}) \\
\text { Waist: hip ratio }\end{array}$ & $\begin{array}{c}65.73(2.52) \\
76.5(7.6) \\
0.81(0.03)\end{array}$ & $\begin{array}{c}64.68(2.67) \\
71.4(7.6)^{*} \\
0.79(0.03)\end{array}$ & $\begin{array}{c}66.10(2.74) \\
73.4(7.4)^{+\neq} \\
0.83(0.04)\end{array}$ & $\begin{array}{c}64.43(2.78) \\
64.4(8.5) \\
0.78(0.01)\end{array}$ & $\begin{array}{c}64.42(2.74) \\
64.3(8.4) \\
0.77(0.01)\end{array}$ & $\begin{array}{c}65.59(2.76) \\
65.7(8.4) \\
0.79(0.01)\end{array}$ \\
\hline
\end{tabular}

Values are mean(s.e.m.); *significantly different from base line; ${ }^{\dagger}$ significantly different from 3 months; ${ }^{\ddagger}$ response over time significantly different from controls, $P<0.05$

Table 2. Serum lipid and lipoprotein variables for walkers and controls

\begin{tabular}{|c|c|c|c|c|c|c|}
\hline & \multicolumn{3}{|c|}{ Walkers $(n=10)$} & \multicolumn{3}{|c|}{ Controls $(n=10)$} \\
\hline & Base line & 12 weeks & 24 weeks & Base line & 12 weeks & 24 weeks \\
\hline Total cholesterol (mmol I-1) & $5.32(0.25)$ & $4.87(0.28)$ & $4.77(0.28)$ & $4.94(0.34)$ & 4.85(0.29) & $4.74(0.29)$ \\
\hline HDL cholesterol $\left(\mathrm{mmol} \mathrm{I}^{-1}\right)$ & $1.21(0.06)$ & $1.27(0.07)^{*}$ & $1.19(0.06)^{t \neq}$ & $1.22(0.06)$ & $1.18(0.05)$ & $1.19(0.06)$ \\
\hline $\mathrm{HDL}_{2}$ cholesterol $\left(\mathrm{mmol} \mathrm{I}^{-1}\right)$ & $0.42(0.02)$ & $0.50(0.02)^{*}$ & $0.48(0.03)^{t \neq}$ & $0.46(0.03)$ & $0.44(0.04)$ & $0.46(0.04)$ \\
\hline Triacylglycerol $\left(\mathrm{mmol} \mathrm{I}^{-1}\right)$ & $0.97(0.13)$ & $0.97(0.16)$ & $0.95(0.17)$ & $0.77(0.08)$ & $0.77(0.06)$ & $0.89(0.12)$ \\
\hline Apo B $\left(\mathrm{g}^{-1}\right)$ & $0.75(0.05)$ & $0.73(0.05)$ & $0.79(0.06)$ & $0.64(0.05)$ & $0.63(0.04)$ & $0.67(0.05)$ \\
\hline
\end{tabular}

Values are mean(s.e.m.); ${ }^{*}$ significantly different from base line; ${ }^{\dagger}$ significantly different from 3 months; ${ }^{\ddagger}$ response over time significantly different from controls, $\boldsymbol{P}<\mathbf{0 . 0 5}$

Table 3. Average daily intake of energy, pecentages of energy from fat and carbohydrate (CHO), ratio of intake of polyunsaturated to saturated fat $(P: S)$ and alcohol for walkers and controls

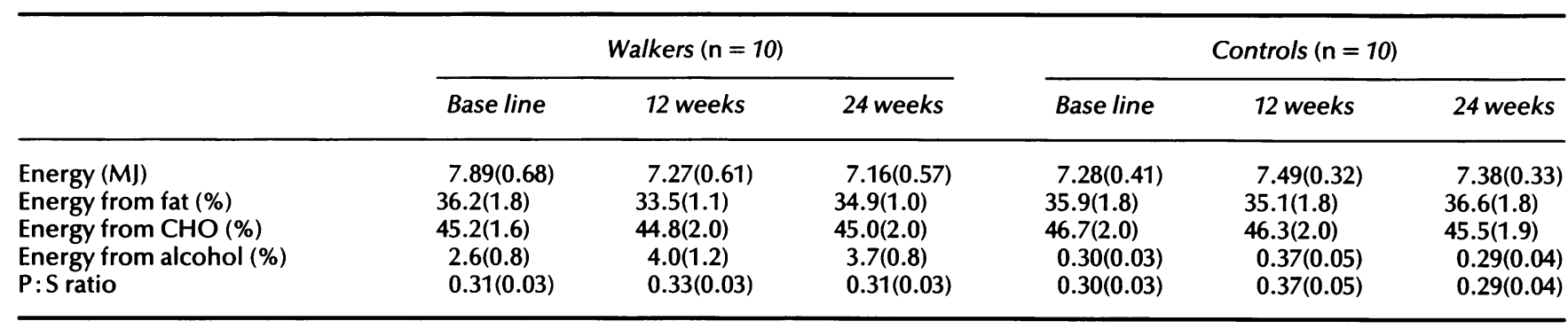

Values are mean(s.e.m.) 
Thus our findings confirm, using laboratory measures of endurance fitness, earlier reports that, given appropriate initial counselling and instruction, formerly sedentary women will adhere to a flexible, home-based, moderate intensity exercise programme ${ }^{17}$. It is noteworthy that the total time invested in brisk walking (maximum of under $5 \mathrm{~h}$ per week), although at first glance a lot, was modest in relation to that needed to travel to, and take part in, an organized exercise class on the often recommended thrice-weekly basis.

Oxygen uptake at a reference blood lactate concentration proved the most sensitive index of the effects of varying physical activity levels, increasing by $30 \%$ with training and decreasing by $11 \%$ when physical levels dropped. This is consistent with rapid changes in the activities of enzymes which are markers for the oxidative capacity of skeletal muscle as a training stimulus is applied and then withdrawn ${ }^{18}$. Brisk walking did provoke a decrease in heart rate but this was not entirely reversed with detraining, possibly because of some residual increase in maximal oxygen uptake; training-induced increases in maximal oxygen uptake can persist for up to 12 weeks after training ceases ${ }^{18}$ and a given bout of exercise may therefore have represented a somewhat lower relative exercise intensity than at base line, even after detraining. Another reason for the difficulty of identifying a detraining effect using heart rate is that baseline values are inevitably somewhat elevated due to an arousal effect. These observations should be borne in mind when using heart rate as an index of a training effect in the absence of a control group.

At least one observational study suggests that women who are physically active, but not endurance trained, possess more favourable blood lipid profiles than sedentary women ${ }^{19}$. Information specifically about walking is more limited but women who walk regularly have been reported to have a markedly lower likelihood of having a TC/HDL-C ratio greater than 5.0 (Reference 20). Furthermore, older women (70 years of age) who walk for at least $30 \mathrm{~min}$ daily are reported to show lower plasma triacylglycerol concentrations than women who do less walking ${ }^{21}$.

Despite these cross-sectional observations, the effectiveness of exercise programmes based on walking in modifying serum lipids and lipoproteins in formerly sedentary women is unclear. Two studies involving walking/slow jogging have found no changes in middle-aged women ${ }^{8,22}$ and two explanations have been advanced: (1) that hypoestrogenic women may be insensitive to the stimulating effects of exercise on HDL cholesterol ${ }^{8}$; and (2) that such moderate exercise does not reach the 'threshold' level of exercise needed to be effective in this regard 8,23 . However, the first explanation is not supported by the increase in HDL cholesterol reported for 68-yearold women following an 8-week walking programme ${ }^{5}$ and the second is not supported by the findings of a 6-month randomized trial involving three different intensities of walking, i.e. $4.8 \mathrm{~km} \mathrm{~h}^{-1}, 6.4 \mathrm{~km} \mathrm{~h}^{-1}$ and $8.0 \mathrm{~km} \mathrm{~h}^{-1}$ (Reference 24). HDL cholesterol increased by a similar amount, relative to controls, in each group, despite the greater improvements in fitness with faster walking speeds. Our findings agree with those of Duncan and co-workers ${ }^{24}$, for women walking briskly at $1.76 \mathrm{~m} \mathrm{~s}^{-1}\left(6.34 \mathrm{~km} \mathrm{~h}^{-1}\right)$ and confirm our earlier findings ${ }^{9}$.

Most of the increase in HDL with walking appeared to be attributable to changes in the $\mathrm{HDL}_{2}$ subfraction, that which differs most markedly between groups in case-control studies ${ }^{25}$, suggesting that brisk walking is associated with improvements in reverse cholesterol transport in these women. The lack of a complementary decrease in triacylglycerol is surprising because the concentration of HDL cholesterol is dependent on the metabolism of triacylglycerol-rich lipoproteins ${ }^{26,27}$. However, Duncan and co-workers also found an increase in HDL cholesterol without a corresponding change in triacylglycerol ${ }^{24}$ and the reason, exacerbated when only a small number of subjects are studied, may be that fasting concentrations may be an insensitive method for examining the metabolic capacity for triacylglycerol ${ }^{28}$.

There is known to be an inverse relationship between body fatness and HDL cholesterol concentrations in women ${ }^{29}$. In the present study, significant changes in subcutaneous fat occurred with changing levels of brisk walking. Controlling for these - by analysis of covariance - suggested that they might be related to the changes in HDL metabolism which were observed. However, local adaptations in skeletal muscle ${ }^{30}$ probably also play a part as HDL cholesterol has been reported to increase with increased physical activity in women even in the absence of changes in adiposity ${ }^{9,25}$.

The magnitude of the changes in HDL cholesterol in these women are modest but the finding still has important implications for public health policy. Data from the Helsinki Heart Study indicate that every 1\% increase may decrease coronary heart disease risk by as much as $3 \%^{31}$. Furthermore, in women, HDL cholesterol concentration has been shown to be a stronger risk factor than LDL cholesterol ${ }^{1}$. In this country, about eight of ten women are physically inactive $^{32}$ and consequently the potential to decrease morbidity and mortality by persuading women to take more exercise may be considerable. Brisk walking carries a low risk of injury and is more likely to be adopted as a long-term exercise habit than exercise of a higher intensity ${ }^{33}$. Furthermore, exercise may act synergistically with other intervention strategies. Wood and co-workers ${ }^{34}$ examined the effects of adding exercise to dietary changes which included a decreased intake of saturated fat and cholesterol. In women, only the group who exercised as well as dieting avoided the decrease in HDL cholesterol which accrues from increasing the proportion of dietary energy obtained from carbohydrate.

Finally, it should be noted that, in women as well as in men, higher levels of physical fitness are, independent of other major risk factors, associated with delayed all-cause mortality and in particular with lower death rates from cardiovascular disease and cancer ${ }^{35}$. Thus the improvements in physical fitness which resulted from brisk walking may be as important in the context of the risk of CHD as the increases in HDL cholesterol concentrations which accompanied them. 


\section{Acknowledgements}

The authors thank David Vallance, Department of Chemical Pathology and Human Metabolism, Royal Free Hospital Medical School, London, UK, for the analysis of apolipoproteins.

\section{References}

1 Bush TL, Fried LP, Barrett-Connor E. Cholesterol, lipoproteins, and coronary heart disease in women. Clin Chem 1988; 34: B60-B70.

2 Kannel WB. Metabolic risk factors for coronary heart disease in women: perspective from the Framingham Study. $A m$ Heart J 1987; 114: 413-19.

3 Brunner D, Weisbort J, Meshulam N, Schwartz S et al. Relation of serum total cholesterol and high-density lipoprotein cholesterol percentage to the incidence of definite coronary events: twenty-year follow-up of the Donolo-Tel Aviv prospective coronary artery disease study. Am J Cardiol 1987; 59: 1271-6.

4 Moore CE, Hartung GH, Mitchell RE, Kappus CM, Hinderlitter J. The relationship of exercise on high-density lipoprotein cholesterol levels in women. Metabolism 1983; 32: 189-96.

5 Whitehurst M, Menendez E. Endurance training in older women. Lipid and lipoprotein responses. Physician and Sportsmedicine 1991; 19: 95-103.

6 Brownell KD, Bachorik PS, Ayerle RS. Changes in plasma lipid and lipoprotein levels in men and women after a program of moderate exercise. Circulation 1982; 65: 477-84.

7 Frey MAB, Doerr BM, Laubach LL, Mann BL, Glueck CJ. Exercise does not change high-density lipoprotein cholesterol in women after 10 weeks of training. Metabolism 1982; 31: 1142-6.

8 Boyden TW, Pamenter RW, Rotkis TC, Morrison DA et al. Effects of exercise training on plasma cholesterol, highdensity-lipoprotein cholesterol, and sex steroid concentrations in women. In Eaker ED, Packard B, Wenger NK, Clarkson TB, Tyroler HA, eds. Coronary Heart Disease in Women. New York, USA: Haymarket Doyma, 1987: 158-63.

9 Hardman AE, Jones PRM, Norgan NG, Hudson A. Brisk walking improves endurance fitness without changing body fatness in previously sedentary women. Eur J Appl Physiol 1992; 65: 345-9.

10 Maritz JS, Morrison JF, Peter J, Strydom NB, Wyndham CH. A practical method of estimating an individual's maximal oxygen intake. Ergonomics 1961: 4: 97-122.

11 Maughan RJ. A simple, rapid method for the determination of glucose, lactate, pyruvate, alanine, 3-hydroxybutyrate and acetoacetate on a single $20 \mu \mathrm{l}$ blood sample. Clin Chim Acta 1982; 122: 231-40.

12 Farish E, Fletcher CD. A comparison of two micro-methods for the determination of $\mathrm{HDL}_{2}$ and $\mathrm{HDL}_{3}$ cholesterol. Clin Chem Acta 1983; 129: 221-8.

13 Warnick GR, Albers JJ. A comprehensive evaluation of the heparin-manganese precipitation procedure for estimating high density lipoprotein cholesterol. J Lipid Res 1978; 19: 65-76.

14 Jones PRM, Hunt MJ, Brown TP, Norgan NG. Waist-hip circumference and its relationship to age and overweight in British men. Human Nutrition: Clinical Nutrition 1986; 40C: 239-47.

15 Paul AA, Southgate DAT. McCance and Widdowson's 'The composition of foods'. London, UK: HMSO, 1978.
16 American College of Sports Medicine. Guidelines for Exercise Testing and Prescription, 4th ed. Philadelphia, USA: Lea and Febiger, 1991: 100.

17 Rogers F, Juneau M, Taylor CB, Haskell WL et al. Assessment by a microprocessor of adherence to home-based moderateintensity exercise training in healthy, sedentary middle-aged men and women. Am J Cardiol 1987; 60: 71-5.

18 Henriksson J, Reitman JS. Time course of changes in human skeletal muscle succinate dehydrogenase and cytochrome oxidase activities and maximal oxygen uptake with physical activity and inactivity. Acta Physiol Scand 1977; 99: 91-7.

19 Haskell WL, Taylor HL, Wood PD, Schrott H, Heiss G. Strenuous physical activity, treadmill exercise test performance and plasma high-density lipoprotein cholesterol. Circulation 1980; 62 (Suppl IV) IV-53-IV-61.

20 Tucker LA, Friedman GM. Walking and serum cholesterol in adults. Am J Public Health 1990; 80: 1111-3.

21 Frändin K, Grimby G, Mellstrom D, Svanborg A. Walking habits and health-related factors in a 70-year-old population. Gerontology 1991; 37: 281-8.

22 Juneau M, Rogers F, de Santos V, Yee M et al. Effectiveness of self-monitored, home-based, moderate-intensity exercise training in middle-aged men and women. Am J Cardiol 1987; 60: 66-70.

23 Superko HR. Exercise training, serum lipids, and lipoprotein particles: is there a change threshold? Med Sci Sports Exerc 1991; 23: 677-85.

24 Duncan JJ, Gordon NF, Scott CB. Women walking for health and fitness. How much is enough? JAMA 1991; 266: 3295-9.

25 Miller NE. Associations of high-density lipoprotein subclasses and apolipoproteins with ischemic heart disease and coronary atherosclerosis. Am Heart J 1987; 113: 589-97.

26 Tall AR. Plasma high density lipoproteins: metabolism and relationship to atherogenesis. J Clin Invest 1990; 86: 379-84.

27 Miesenböck G, Patsch JR. Relationship of triglyceride and high density lipoprotein metabolism. Atherosclerosis Rev 1990; 21: 119-27.

28 Patsch JR, Miesenböck G, Hopferwieser T, Mühlberger V et al. Relation of triglyceride metabolism and coronary artery disease. Studies in the postprandial state. Arteriosclerosis Thrombosis 1992; 12: 1336-45.

29 Manson JA, Colditz GA, Stampfer MJ, Willett WC et al. A prospective study of obesity and risk of coronary heart disease in women. New Eng J Med 1990; 322: 882-9.

30 Kiens B, Lithell $\mathrm{H}$. Lipoprotein metabolism influenced by training-induced changes in human skeletal muscle. J Clin Invest 1989; 83: 558-64.

31 Manninen V, Elo MO, Frick MH, Haapa $\mathrm{K}$ et al. Lipid alterations and decline in the incidence of coronary heart disease in the Helsinki Heart Study. IAMA 1988; 260: 641-51.

32 National Fitness Survey. Report. London, UK: Health Education Council and Sports Council, 1992.

33 Martin JE, Dubbert PM. Exercise applications and promotion in behavioural medicine: current status and future directions. Consulting Clin Psychol 1982; 50: 1004-17.

34 Wood PD, Stefanick ML, Williams PT, Haskell WL. The effects on plasma lipoproteins of a prudent weight-reducing diet, with or without exercise, in overweight men and women. N Engl J Med 1991; 325: 461-6.

35 Blair SN, Kohl HW, Paffenbarger RS, Clark DG, Cooper KH, Gibbons LW. Physical fitness and all-cause mortality. A prospective study of healthy men and women. JAMA 1989; 262: 2395-401. 